\title{
A Hybrid Approach of Using Particle Swarm Optimization and Volumetric Active Contour without Edge for Segmenting Brain Tumors in MRI Scan
}

\author{
Ali M. Hasan \\ Al-Nahrain University- College of Medicine- Baghdad, Iraq
}

\section{Article Info \\ Article history: \\ Received Jul 20, 2018 \\ Revised Aug 5, 2018 \\ Accepted Sep 6, 2018}

\section{Keyword:}

Active contour without edge Magnetic resonance imaging Particle Swarm Optimization

\begin{abstract}
Segmentation of brain tumors in magnetic resonance imaging is a one of the most complex processes in medical image analysis because it requires a combination of data knowledge with domain knowledge to achieve highly results. Such that, the data knowledge refers to homogeneity, continuity, and anatomical texture. While the domain knowledge refers to shapes, location, and size of the tumor to be delineated. Due to recent advances in medical imaging technologies which produce a massive number of cross-sectional slices, this makes a manual segmentation process is a very intensive, timeconsuming and prone to inconsistences. In this study, an automated method for recognizing and segmenting the pathological area in MRI scans has been developed. First the dataset has been pre-processed and prepared by implementing a set of algorithms to standardize all collected samples. A particle swarm optimization is utilized to find the core of pathological area within each MRI slice. Finally, an active contour without edge method is utilized to extract the pathological area in MRI scan. Results reported on the collected dataset includes 50 MRI scans of pathological patients that was provided by Iraqi Center for Research and Magnetic Resonance of Al Imamain Al-Kadhimain Medical City in Iraq. The achieved accuracy of the proposed method was $92 \%$ compared with manual delineation.
\end{abstract}

Copyright $\left({ }_{0} 2018\right.$ Institute of Advanced Engineering and Science. All rights reserved.

\section{Corresponding Author:}

Ali M. Hasan,

Al-Nahrain University-College of Medicine-Baghdad, Iraq.

Email: a.hasan4@edu.salford.ac.uk

\section{INTRODUCTION}

Over the last two decades, the rapid development of non-invasive imaging technologies has led to increase the number of slices that are produced for each patient, this is becoming a time consuming and slow process that is also prone to errors [1]. The diagnosis of these slices has become tedious and complex process for clinicians who have to have high experience to segment them manually. To address these difficulties, computerized image segmentation is required and it has taken a vital role in medical imaging technologies due to the diversity in object shapes and heterogeneity in intensity [2].

Tumors of nervous system are relatively unfamiliar compared with other neoplasm. They produce symptoms and signs due to direct tissue damage. These symptoms associate with tumor location [3]. Brain tumors require special studies because they are complicated pathology, making them hard to diagnose, different histologic and topographical distribution [3]. Generally, surgery is considered as the first step that should be taken to treat and achieve a gross total resection (GTR) of brain tumors. It is the preferable treatment when a tumor could be removed without any risks and side effects to the brain [4]. While radiotherapy and chemotherapy are used to slow the growth of brain tumors that cannot be removed by surgery [5]. The two most important and popularly used imaging technologies are computed tomography (CT) and magnetic resonance imaging (MRI). MRI is the best medical imaging technology for diagnosing 
pathological brains by providing details MR images from different modelities. These MR images can be used to study and compare brain tumors on preoperative and postoperative, in addition to be used to determine the resection extent $[4,6]$. The most commonly standard MRI protocols are T1-weighted images (T1W), T2weighted images (T2W) and fluid attenuated inversion recovery (FLAIR) and T1W images with contrast enhancement (T1CW) [7]. Moreover, MRI is highly sensitive to the any variations in tissue density. Most of brain tumors appears as hypo-intense compared to the surrounded normal tissue on T1W modality and appears as hyper-intense on T2W modality [1]. Accordingly, T2W slices are considered initially to give an initial evaluation, tumor grade identification and recognizing pathological area from normal tissues. However, some tumors appear heterogeneously in MRI scan due to presence of fat, hemorrhage and necrosis. Therefore, utilizing of contrast material is essential to distinguish the boundaries of some brain tumors from normal adjacent tissue [7]. Analysis of these diverse types of MRI images requires advanced computerized quantification and visualization tools and this could be supported by digital image processing technology. Image processing is concerned with the manipulation and interpretation of the objects in digital images by removing the effect of noise that is acquired during the acquisition process, deriving features and properties that could be used to discriminate the pathological patients and assign the core of abnormalities in the brain scans.

The expansion of image processing to different fields of application has associated with the recent progress of medical imaging technologies, where more acquisition modelities and more images are produced. This makes that the visualization of the acquired images alone is insufficient without image processing applications. Such that, image processing applications have a significant impact on the diagnosis process by helping the clinician to analyze all volumetric data to make a therapeutic decision and predict outcomes of disease. Over the last few decades, there were a massive number of studies that covered the topic of brain tumor segmentation because it is an important to understand information that is obtained by MRI for diagnosis and monitoring the effectiveness of treatment [8]. Soltaninejad, et al. [9] developed a new system for detection and segmentation of brain tumors automatically using FLAIR images. This method was based on using super-pixel technique and classification of each super-pixel. From edge super-pixel within FLAIR image, a set of features were extracted including intensity-based, Gabor wavelet, fractal analysis and curvatures. The extracted features were classified by Extremely randomized trees (ERT) and the determined dice score was $88 \%$ for BRATS 2013 dataset. Lee, et al. [10] developed a method for segmentation of volumetric medical scans based on using the geometric deformable model and level set method. Such that, robust alignment, active region and smoothing measures were derived from the calculus of variation principle to manage the speed function in level set approach to reach the boundaries of the target object without any boundary leakage problem. The achieved Jaccard's measure score was (72.5\%-94.1\%). Hasan, et al. [1] proposed a fully automated system for classifying and segmenting MRI brain scan. The proposed system was capable to assign the core of pathological area within brain scan and this helped to initialize and start the segmentation process automatically. Volumetric segmentation based on three-dimensional active contour without edge (3DACWE), was used to segment a full MRI scan. The achieved dice score was $89.3 \%$ for BRATS 2013 dataset. Gordillo, et al. [11] concluded that it is hard to achieve good results of segmenting brain tumors by using the conventional methods such as thresholding, region growing and edge detection techniques. In addition to appear cross-sectional imaging technologies, this makes a segmentation task is a challenging problem to detect object boundaries across set of slices. Thapaliya, et al. [12] proposed a new method based on using level set method for segmenting brain tumors in MRI scans. The author initialized the level set method by using a new sign pressure function (SPF) instead of using signed distance function (SDF) to avoid of moving the zero level set away from its interface exists. The SPF method uses the adaptive local statistics features of the desired object in MRI scan.

Active contour models are highly significant and have used widely for brain tumor segmentation. This approach is distinctively used for distinguishing the margins between the pathological area and the surrounding normal tissue based on deriving conditions from the biological and anatomical knowledge [13]. Active contour models work under influence of two forces (internal and external forces). These two forces manage pushing and pulling of contour toward the required object's boundaries [1]. The main problem of active contour models is the determination of initial starting point. To overcome this problem, we develop a new method to choose the point that the contour starts from, this method exploits the prior knowledge that the normal brain structure is symmetry around the mid-sagittal plane of brain. The proposed method searches in parallel for the most various area in MRI scan which represents the pathological area in the brain.

The rest of this paper is ordered as follows. Section 2 demonstrates the details of the proposed method. Section 3 presents and discusses the experimental results. Finally the conclusion is presented in Section 4. 


\section{RESEARCH METHOD}

In this paper, an automated segmentation method of brain tumors is developed, which consists of two essential steps; image preprocessing and bounding cubes based particle swarm optimization (BCBPSO).

\subsection{Image Preprocessing}

The collected images from medical scan centers are not suitable for direct processing due to acquired noise that could contaminate these images during the acquisition or transformation process, intrascan and inter-scan image intensity variations between the consecutive MRI slices and between different scanners even used identical acquisition protocols. In addition to the variation in the intensity can vary dramatically due to different scanner manufacturers, models, bias field and different pulse sequence parameters [14-16]. The preprocessing analysis is considered an important step in all automated classification and segmentation systems. Typically, it includes image enhancement and intensity normalization. Image enhancement techniques can be used to increase the perceptibility of objects in images to the human spectator and for further automatic analysis.

Magnetic resonance (MR) images are usually corrupted by random noise from the acquisition process, and it generates a small random changes in the intensity of pixels. These modifications may be lead to erroneous segmentation and feature extraction. Therefore, noise removing algorithms have been used to improve image quality [17-20]. In this study, the two-dimensional Bilateral filtering is used to denoise the MRI images. The basic idea of Bilateral filtering is to weight the coefficients of filter with their associated relative pixel intensities to smooth images with edge-preserving. It is a non-iterative image smoothing scheme for edge-preserving by combined non-linearly nearby pixels [17]. The principle of Bilateral filtering is that every two pixels are close to each other if they occupy nearby spatial locations and have some similarity in the photometric range. The Bilateral filtering expression is given in Equation 1 [21]:

$$
B F[I]_{P}=\frac{1}{W_{P}} \sum_{q \in S} G_{\sigma_{S}}(\|P-q\|) G_{\sigma_{r}}\left(\left\|I_{P}-I_{q}\right\|\right) I_{q}
$$

where $W_{P}$ denotes a normalization factor and given Equation 2:

$$
\mathrm{W}_{\mathrm{P}}=\sum_{\mathrm{q} \in \mathrm{S}} \mathrm{G}_{\sigma_{\mathrm{S}}}(\|\mathrm{P}-\mathrm{q}\|) \mathrm{G}_{\sigma_{\mathrm{r}}}\left(\left\|\mathrm{I}_{\mathrm{P}}-\mathrm{I}_{\mathrm{q}}\right\|\right)
$$

where $\sigma_{S}$ and $\sigma_{r}$ measure the amount of filtering for the given image $I . G_{\sigma_{S}}$ denotes a spatial Gaussian that reduces the impact of distance pixels, $G_{\sigma_{r}}$ denotes a range Gaussian that reduces the impact of pixels $\mathrm{q}$ with the intensity value different from $I_{P}$. Figure 1 shows an example of implementing Bilateral filtering on the given noisy MRI slice (Figure 1(A)), and the output of filter is shown in Figure 1(B).

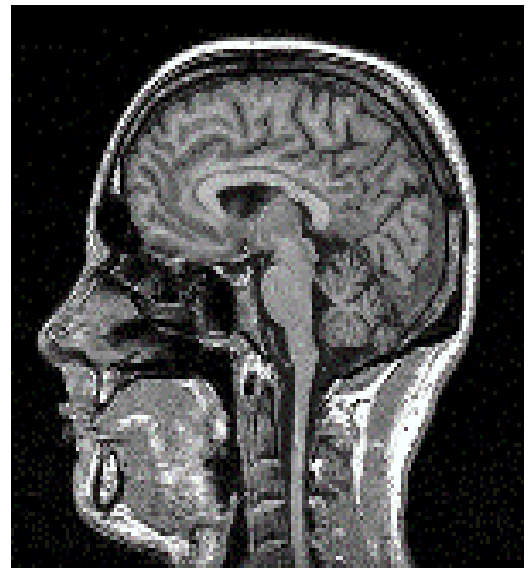

(a)

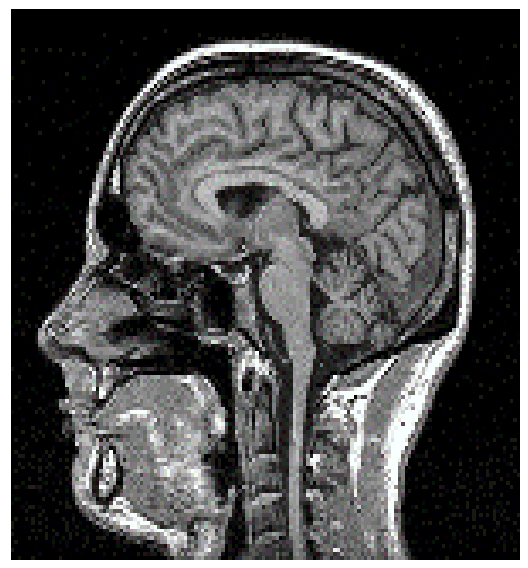

(b)

Figure 1. The output of bilateral filtering, a) Original noisy MRI slice, b) Denoised MRI slice 
Intensity normalization is an important preprocessing step in the study and analysis of medical cross-sectional imaging technology. Such that, the texture of the produced slices is highly sensitive to the acquisition setting (e.g. MR protocols, scanner brand, and MR adjustment). This acquires undesired variation in intensity which may impact on the outcome of MRI images analysis [22]. Therefore, intensity normalization and standardization of MRI slices is essential to enhance image compatibility by reducing the intensityvariabililtywhich is produced by different setting, different radiologists and different equipment, and this may have a considerable influence on the clinical diagnosis [13]. According to Hasan, et al. [1], Loizou, et al. [22], Tantisatirapong [14], and Nabizadeh [23] the histogram normalization method has been chosen as a best normalization techniques. Such that, it stretches and shifts the histogram of the given MRI slice to cover all the grey scale levels.

\subsection{Bounding Cubes Based Particle Swarm Optimization (BCBPSO)}

The bounding cubes based particle swarm optimization (BCBPSO) method is proposed to recognize the core of the most dissimilar area in brain without need for clinician. The input is a volumetric MRI scan of a single patient and the output is a subset of pathological MRI slices. The basic idea of this method is based on hemispheric symmetry of normal brain around the mid-sagittal plain (MSP) of brain. Where, the most anatomical tissues in left hemisphere of the brain has a counterpart on the right hemisphere of the brain at same location and orientation.

These hemispheres are splitted by a longitudinal fissure that can be used as a reference for asymmetry analysis and represents the main landmark and good indicator about the brain normality or abnormality such that most pathologies (e.g. tumors, strokes and bleeding) can be diagnosed by analyzing a symmetry of MRI brain scan [13, 24]. However, the symmetry of brain can be destroyed by growing tumor cells that may curve the MSP of the brain [25]. There are many segmentation algorithms, were proposed but they were not completely automated. These algorithms require user interaction to select the initial seed point to start the segmentation process. Since the main characteristics that can be used to detect the brain tumors is the variation in the intensity levels of the MRI brain slice between both hemispheres of brain. BCBPSO method requires implementing some preprocessing to correct MSP of brain if there is any skewness in the patient's head. In addition to compress the low-contrast and expand the high-contrast regions by using exponential transforming.

\subsubsection{MSP of Brain Identification}

The MSP identification is necessary and important step in diagnosing and analyzing brain scans because of considering this plane gives an initial prediction of brain's pathology [26]. Due to the MSP of brain matches with the patient's head orientation, the orientation will be used in this paper to assign the MSP of the brain. Where, all MRI axial slices have inter-hemispheric fissure lines are parallel with the same orientation of the patient's head [13]. Principle component analysis method is used to locate the orientation of patient's head and measure the degree of skewness of MSP of the brain as explained and described in details by Hasan and Meziane [13].

\subsubsection{Exponential Transformation of MRI Slices' Intensity}

Because brain tumors appear brighter than the surrounding normal tissues in MRI T2 weighted slices, as well as the BCBPSO method searches about a region that includes a significant intensity variation between both hemispheres of brain. Thus, an exponential transformation is implemented prior to BCBPSO to expand the brighter regions and compress the dark region nonlinearly. Subsequent, the intensity differences between the pathological region and the surrounding normal tissue would be increased [13, 27]. Resulting in faster and precisely converging to the pathological region of the brain. Figure 2 demonstrates an example of implementing exponential transformation on MRI slice from BRATS 2013 dataset.

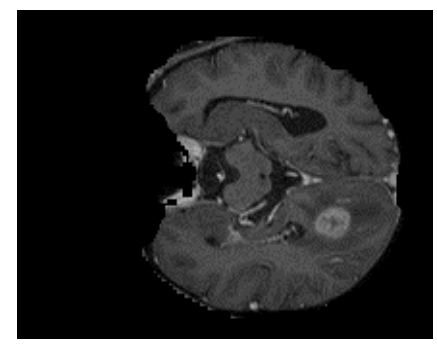

(a)

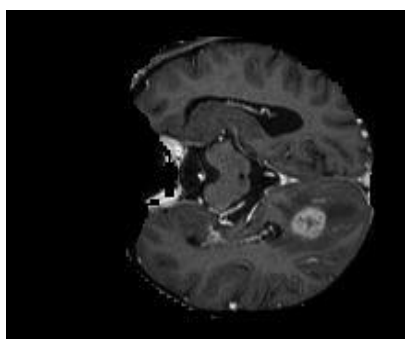

(b)

Figure 2. Image exponential transformation, A) Original MRI brain slice, B) Transformed MRI brain slice

A Hybrid Approach of Using Particle Swarm Optimization and Volumetric Active Contour... (Ali M. Hasan) 


\subsubsection{Particle Swarm Optimization (PSO)}

PSO is a population-based computational intelligence technique, proposed to solve optimization problems [28]. The populations of PSO handle a set of randomly initialized solutions known as swarm that include a number of particles, each one represents potential solutions to the optimization task and moves iteratively through hyperspace to new positions according to the velocity equation as given in Equation 3 . This technique is inspired from social behavior of bird flocking where all the particles are guided by the best particle of the swarm and each particle keeps tracking of its best solution found through iterations. This merit represents the main advantage with regard to other evaluatationally computation techniques. Additionally, PSO is not computationally expensive [28].

$$
v_{i}(n+1)=\varphi v_{i}(n)+K r_{1}\left(P_{\text {best }}-x_{i}(n)\right)+K r_{2}\left(P_{\text {gbest }}-x_{i}(n)\right)
$$

where $v_{i}$ represents the current velocity of the individual $x_{i}$ at $\mathrm{n}$ time step, $\varphi$ is the inertia weight, $\mathrm{K}$ denotes the learning factor, $P_{\text {best }}$ represents current best solution found by the current individuals, $P_{\text {gbest }}$ represents the best solution found by the whole swarm. $r_{1}$ and $r_{2}$ are two constants and are distributed uniformly [28]. After updating the velocity, the new position of individual is computed by using Equation 4:

$$
x_{i}(n+1)=x_{i}(n)+v_{i}(n+1)
$$

Updating of velocity and moving of individuals continue until stopping criteria is satisfied. BCBPSO is based on searching and identifying the location of the most dissimilar area between both hemispheres of the brain automatically. It exploits the prior knowledge that the similarity between hemispheres of brain is reduced with appearing of brain tumors. The proposed method is based on generating randomly a hundred of volumetric small cubes with same sizes. These cubes are moved periodically within iterations of PSO toward the pathological area that increased the value of the objective function [29]. The objective function value is increased when the generated cube stands on the pathological area and decreased when standing on soft tissue because the tumors tissue is always inhomogeneous and brighter that the surrounding normal tissue [29]. Each generated cube is compared with the opposite cube in other side of brain. The objective function $G$ was suggested by Hasan [29], and is used in this paper as given in Equation 5:

$$
G=\frac{1}{x * y * z}\left|\sum_{i, j, k}^{x, y, z} I_{L}(i, j, k)-\sum_{i, j, k}^{x, y, z} I_{R}(i, j, k)\right|
$$

where $x, y$ and $z$ represent the coordinates of the generated cubes in the left $I_{L}$ and right $I_{R}$ hemispheres of brain. Figure 3, illustrates the pseudo-code for the proposed method.

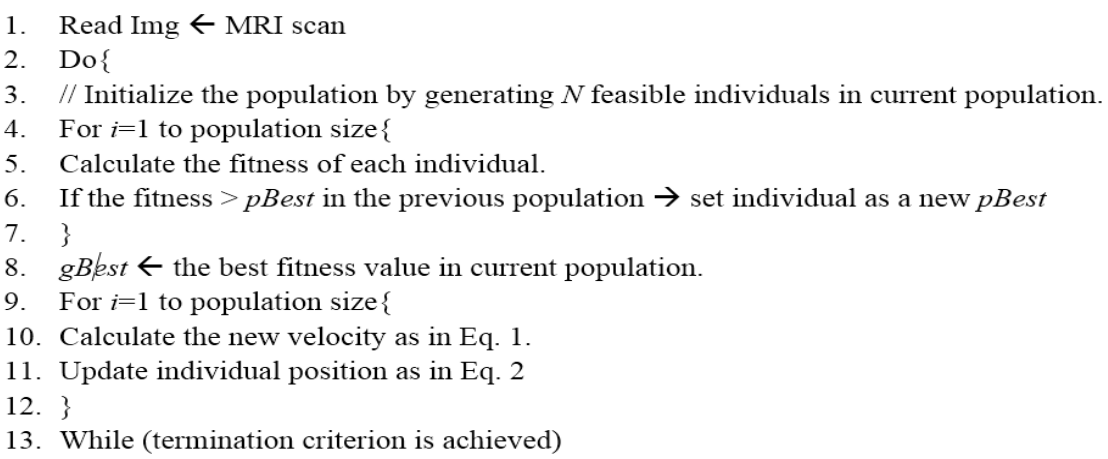

Figure 3. BCBPSO's pseudo-code

\subsection{Volumetric Segmentation of Brain Tumors}

Manual segmentation of brain tumors is considered an exhaustion task and extremely exposed to errors due to the variety of tumors and the difficulty in identifying the tumor location, size and texture. As well as, difficulty of using available methods such as thresholding, region-based and edge based methods in 
segmenting brain tumors [1]. Additionally, appearance of cross-sectional medical imaging data provides additional challenge for segmenting boundaries belonging to the same object. The deformable models has been proved that precisely and successfully retrieve the shape of biological structures in many medical image segmentation applications. They combine the derived constraints from the given image and a prior information about the object's [30]. They are classified into edge-based models and region-based models.

Local edge information is used by Edge-based models to attract contour toward the object boundaries, while region characteristics such as intensity homogeneity in each region is used by region-based models to attract contour toward the object boundaries [31]. One of the most common region-based models is the Chan-Vese active model that is based on level-set and Mumford-Shah functional techniques to satisfying the evolution equation as given in Equation 6:

$$
\mathcal{F}^{M S}(u, C)=\int_{\Omega}(u-I)^{2} d x d y d z+\mu \int_{\Omega / C}|\nabla u|^{2} d x d y d z+v|C|
$$

where $\mathrm{u}$ is a piecewise smooth function, the first expression attracts $\mathrm{u}$ to approach to $\mathrm{I}$ and the second expression ensures that $\mathrm{u}$ is differentiate on $\Omega / \mathrm{C}$, and the third expression is responsible about the regularity on $\mathrm{C}$. $\mathrm{u}$ is supposed to be constant in each enclosed area in order to avoid the complexity of implementing the Mumford and Shah function, $u$ is supposed to be had only two values according to Chan and Vese as given in Equation 7 [32]:

$$
u(x, y, z)=\left\{\begin{array}{c}
C_{1} \text { where } x, y, z \text { are inside } C \\
C_{2} \text { where } x, y, z \text { are outside } C
\end{array}\right.
$$

where $\mathrm{C}$ represents the contour, $\mathrm{c} 1$ represents the value of $\mathrm{u}$ inside the contour $\mathrm{C}$, and $\mathrm{c} 2$ represents the value of $\mathrm{u}$ outside the contour $\mathrm{C}$. The Chan-Vese proposed an energy function that is given in Equation 8 [32-34]:

$$
\begin{array}{r}
\mathcal{F}^{C V}\left(C, c_{1}, c_{2}\right)=\mu \text { Length }(C)+v \text { Area }(\text { inside }(C)) \\
+\lambda_{1} \int_{\text {inside }(C)}\left|I(x, y, z)-c_{1}\right|^{2} d x d y d z \\
+\lambda_{2} \int_{\text {outside }(C)}\left|I(x, y, z)-c_{2}\right|^{2} d x d y d z
\end{array}
$$

where, the first expression is responsible for regularizing $\mathrm{C}$ by penalizing the length, and the second expression is responsible for controlling the size by penalizing the enclosed area of C. $\lambda_{1}, \lambda_{2}, \mu$ and $v \geq 0$ are constant parameters that must be set carefully to minimize the energy function optimally. Where, $\lambda_{1}$ and $\lambda_{2}$ are used to calibrate the internal and external forces respectively. While, $\mu$ is used to control the smoothness of contour C. Meanwhile, $v$ controls the possibility of accept or reject the area inside the contour $\mathrm{C}$. The standard gradient descent method is used to minimize the Chan-Vese energy function (Equation 8), resulting in an optimal contour $\mathrm{C}$ that segment the given image $I$.

\section{RESULTS AND ANALYSIS}

The proposed method is evaluated qualitatively and quantitatively on the collected dataset by Hasan and Meziane [13]. This dataset was provided by Iraqi Center for Research and Magnetic Resonance of $\mathrm{Al}$ Imamain Al-Kadhimain Medical City in Iraq, and included 50 pathological patients with different brain tumors. The BCBPSO method is based essentially on the factor that the pathological area is relatively brighter than the surrounding normal tissues. Figure 4, shows how the particles of PSO are moved within volumetric MRI scan toward the pathological area. Because of swarm size is equivalent to population size in genetic algorithm and the optimal size of population was already proved by Hasan, et al. [29] and it is 100. The optimal swarm size is 100 . 


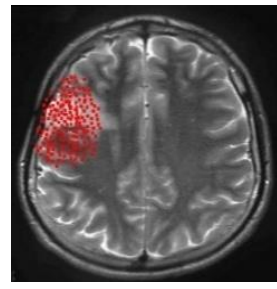

$n=1$

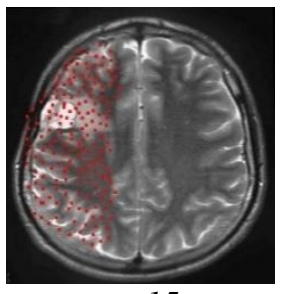

$n=15$

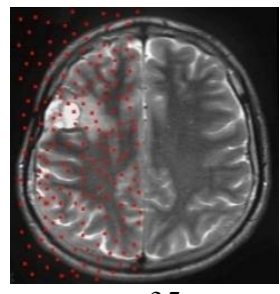

$n=35$

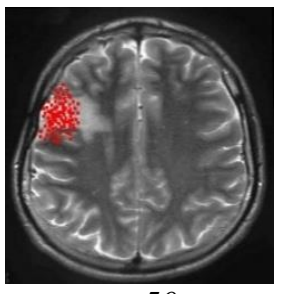

$n=50$

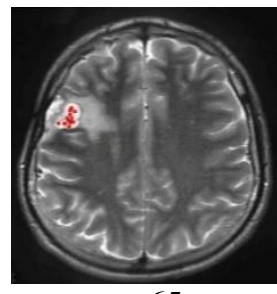

$n=65$

Figure 4. Implementation of BCBPSO on pathological MRI scan with a swarm size equal to 100 . The red rectangles denotes the location of particles in swarm at iterations $(n) 1,15,35,50$ and 65

The BCBPSO method was implemented on 50 MRI scans of pathological brain, and failed to identify location of abnormality of three patients only because those patients have more than one tumor each.After identifying the core of brain tumor of each patient in the given dataset by the BCBPSO method, the volumetric active contour without edge method was initialized and implemented on T2-w MRI modality of given dataset. Figure 5, shows the output of applying the volumetric active contour without edge method to the pathological T2-w MRI brain scan.

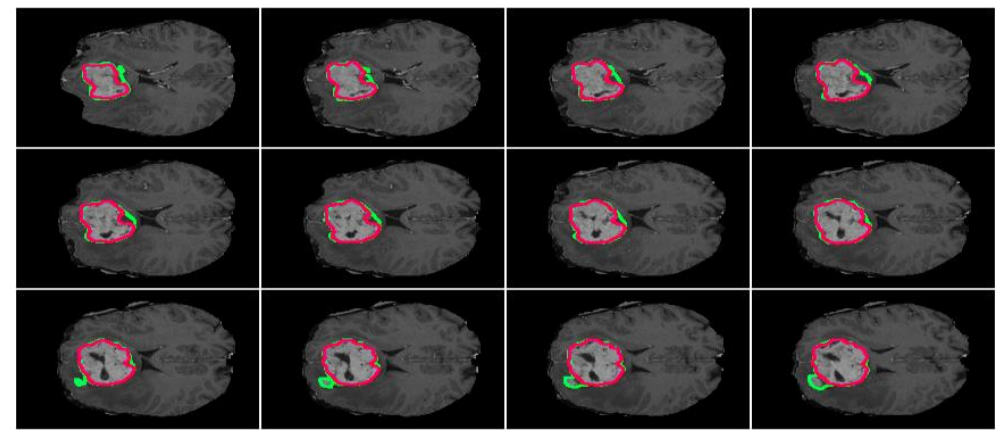

Figure 5. Qualitative outputs of volumetric active contour without edge method on MRI T2-w scan. The green delineation represents the manual segmentation, and the red delineation represents the segmentation by the proposed method

The overall results of the proposed method of four MRI modalities (collected dataset) are shown in Figure 6. The highest segmentation accuracy was achieved in T1c-w modality. While the lowest accuracy was achieved in T2-w modality. A comparison between the achieved results of the proposed method and those achieved by Hasan, et al. [1] is demonstrated in Table 1. It is noted that the three-diemsional segmentation method outperforms the two-dimensional scheme due to exploit the entire volumetric MRI data within the segmentation process instead of segmenting each slice separately.

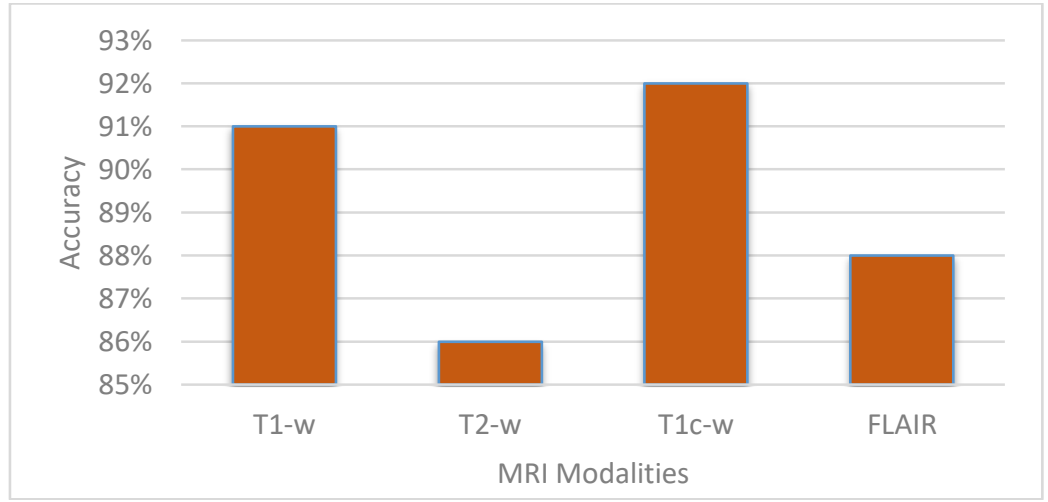

Figure 6. Average accuracy of segmentation of four MRI modalities 
Table 1. Comparison of the Achieved Accuracy with Previous Study

\begin{tabular}{ccccc}
\hline Method & T2-w & T1-w & T1c-w & FLAIR \\
\hline 2DACWE & $83.7 \%$ & $84.4 \%$ & $86.6 \%$ & $82 \%$ \\
Proposed Method & $66.1 \%$ & $91.3 \%$ & $92.2 \%$ & $82.2 \%$ \\
\hline
\end{tabular}

\section{CONCLUSION}

This paper aims to propose a new automated segmentation technique by combining the BCBPSO and volumetric active contour without edge methods. Such that the BCBPSO method was used to assign the core of brain tumors in MRI scans by exploiting the symmetry merit of normal brain scanning. This will help to initialize automatically the volumetric active contour without edge method without need for human. The achieved accuracy shows that the proposed technique outperforms other methods. Recommendation for further research, it is essential to optimize the proposed algorithm with repect to faster implementation in computer network that will run in parallel mode.

\section{REFERENCES}

[1] A. Hasan, F. Meziane, R. Aspin, and H. Jalab, "Segmentation of Brain Tumors in MRI Images Using ThreeDimensional Active Contour without Edge", Symmetry, vol. 8, p. 132, 2016.

[2] C. Xu, D.L. Pham, and J.L. Prince, "Image segmentation using deformable models", Handbook of medical imaging, vol. 2, pp. 129-174, 2000.

[3] G. Karkavelas and N. Tascos, "Epidemiology, Histologic Classification and Clinical Course of Brain Tumors", in Imaging of Brain Tumors with Histological Correlations, A. Drevelegas, Ed., ed: Springer Berlin Heidelberg, 2011, pp. 1-12.

[4] American Brain Tumor Association, "SURGERY", USA, 2015.

[5] M. Havaei, A. Davy, D. Warde-Farley, A. Biard, A. Courville, Y. Bengio, et al., "Brain Tumor Segmentation with Deep Neural Networks", Medical Image Analysis, vol. 35, pp. 18-31, 2017.

[6] H.J. Kim and W. Kim, "Method of tumor volume evaluation using magnetic resonance imaging for outcome prediction in cervical cancer treated with concurrent chemotherapy and radiotherapy", Radiation oncology journal, vol. 30, pp. 70-77, 2012.

[7] A. Drevelegas and N. Papanikolaou, "Imaging modalities in brain tumors", in Imaging of brain tumors with histological correlations, D. Anttabonios, Ed., ed: Springer Berlin Heidelberg, 2011, pp. 13-33.

[8] J. Mikulka and E. Gescheidtov, "An Improved Segmentation of Brain Tumor, Edema and Necrosis", presented at the Progress In Electromagnetics Research Symposium Proceedings,Taipei, 2013.

[9] M. Soltaninejad, G. Yang, T. Lambrou, N. Allinson, T.L. Jones, T.R. Barrick, et al., "Automated brain tumour detection and segmentation using superpixel-based extremely randomized trees in FLAIR MRI", International Journal of Computer Assisted Radiology and Surgery, vol. 12, pp. 183-203, February 012017.

[10] M. Lee, W. Cho, S. Kim, S. Park, and J.H. Kim, "Segmentation of interest region in medical volume images using geometric deformable mode," Computers in biology and medicine, vol. 42, pp. 523-537, 2012.

[11] N. Gordillo, E. Montseny, and P. Sobrevilla, "State of the art survey on MRI brain tumor segmentation," Magnetic Resonance Imaging, vol. 31, pp. 1426-1438, 2013.

[12] K. Thapaliya, J. Pyun, C. Park, and G. Kwon, "Level set method with automatic selective local statistics for brain tumor segmentation in MR images", Computerized Medical Imaging and Graphics, vol. 37, pp. 522-537, 2013.

[13] A. Hasan and F. Meziane, "Automated screening of MRI brain scanning using grey level statistics", Computers \& Electrical Engineering, vol. 53, pp. 276-291, 2016.

[14] S. Tantisatirapong, "Texture analysis of multimodal magnetic resonance images in support of diagnostic classification of childhood brain tumours", $\mathrm{PhD}$, School of Electronic, Electrical and Computer Engineering, University of Birmingham, UK, 2015.

[15] R. Nowak, "Wavelet-based Rician noise removal for magnetic resonance imaging", IEEE Transactions on Image Processing, , vol. 8, pp. 1408-1419, 1999.

[16] E. Anju, M. Karnan, and R. Sivakumar, "MR brain image classification with supervised bacteria foraging technique using SVM", International Journal of Futuristic Science Engineering and Technology, vol. 1, pp. 318-322, , 2013.

[17] Y. Wang, X. Che, and S. Ma, "Nonlinear filtering based on 3D wavelet transform for MRI denoising", EURASIP Journal on Advances in Signal Processing, vol. 2012, pp. 1-14, 2012/02/21 2012.

[18] A. a. Al-Shamasneh, H. Jalab, S. Palaiahnakote, U. Obaidellah, R. Ibrahim, and M. El-Melegy, "A New Local Fractional Entropy-Based Model for Kidney MRI Image Enhancement", Entropy, vol. 20, p. 344, 2018.

[19] R.W. Ibrahim, A.M. Hasan, and H.A. Jalab, "A new deformable model based on fractional Wright energy function for tumor segmentation of volumetric brain MRI scans", Computer Methods and Programs in Biomedicine, vol. 163, pp. 21-28, 2018/09/01/ 2018.

[20] N. Kamaruddin, N.A. Abdullah, and R.W. Ibrahim, "Local region-based ACM with fractional calculus for boundary segmentation in images with intensity inhomogeneity", Malaysian Journal of Computer Science, vol. 29, pp. 124-144, 2016.

[21] S. Paris, P. Kornprobst, J. Tumblin, and F. Durand, "A gentle introduction to bilateral filtering and its applications", in ACM SIGGRAPH 2007 courses, 2007, p. 1. 
[22] C.C. Loizou, M. Pantziaris, I. Seimenis, and C.S. Pattichis, "Brain MR image normalization in texture analysis of multiple sclerosis", presented at the 9th International Conference on Information Technology and Applications in Biomedicine, Larnaca, 2009.

[23] N. Nabizadeh, "Automated Brain Lesion Detection and Segmentation Using Magnetic Resonance Images", PhD, Department of Electrical and Computer Engineering, University of Miami, USA, 2015.

[24] G. Ruppert, L. Teverovskiy, Y. Chen-Ping, X. Falcao, and L. Yanxi, "A new symmetry-based method for midsagittal plane extraction in neuroimages", in International Symposium on Biomedical Imaging: From Nano to Macro, USA, 2011, pp. 285-288.

[25] Y. Liu, R. Collins, and W. Rothfus, "Automatic bilateral symmetry (midsagittal) plane extraction from pathological 3D neuroradiological images", in Procedings of Medical Imaging 1998: Image Processing conference, USA 1998, pp. 1528-1539.

[26] S. Jayasuriya and A. Liew, "Symmetry plane detection in neuroimages based on intensity profile analysis", presented at the International Symposium on Information Technology in Medicine and Education (ITME), Australia, 2012.

[27] M. Khandani, R. Bajcsy, and Y. Fallah, "Automated Segmentation of Brain Tumors in MRI Using Force Data Clustering Algorithm", presented at the In International Symposium on Visual Computing, USA, 2009.

[28] I. Cruz-Aceves, J.G. Aviña-Cervantes, J.M. López-Hernández, and S.E. González-Reyna, "Multiple active contours driven by particle swarm optimization for cardiac medical image segmentation", Computational and mathematical methods in medicine, vol. 2013, 2013.

[29] A. Hasan, F. Meziane, and M. Abd Kadhim, "Automated Segmentation of Tumours in MRI Brain Scans", presented at the Proceedings of the 9th International Joint Conference on Biomedical Engineering Systems and Technologies (BIOSTEC), Rome, Italy, 2016.

[30] H.H. Chang and D.J. Valentino, "An electrostatic deformable model for medical image segmentation", Computerized Medical Imaging and Graphics, vol. 32, pp. 22-35, 2008.

[31] L. Chunming, K. Chiu-Yen, C. Gore, and D. Zhaohua, "Minimization of Region-Scalable Fitting Energy for Image Segmentation", IEEE Transactions on Image Processing, vol. 17, pp. 1940-1949, 2008.

[32] F. Chan and A. Vese, "Active contours without edges", IEEE Transactions on Image Processing, vol. 10, pp. 266$277,2001$.

[33] F. Chan, B. Sandberg, and A. Vese, "Active contours without edges for vector-valued images", Journal of Visual Communication and Image Representation, vol. 11, pp. 130-141, 2000.

[34] F. Chan and A. Vese, "An active contour model without edges", in Scale-Space'99 Corfu, Greece, 1999, pp. 141151. 